\title{
Human Fall Detection Algorithm Design Based on Sensor Fusion and Multi-threshold Comprehensive Judgment
}

\author{
Junsuo Qu, ${ }^{1 *}$ Chen $\mathrm{Wu}^{2}{ }^{2}$ Qian $\mathrm{Li},{ }^{2}$ Ting Wang, ${ }^{2}$ and Abdel Hamid Soliman ${ }^{3}$ \\ ${ }^{1}$ Xi'an Key Laboratory of Advanced Control and Intelligent Process, \\ 618 West Chang'an Street, Chang'an District, Xi'an City 710121, China \\ ${ }^{2}$ School of Communication and Information Engineering, Xi'an University of Posts and Telecommunications, \\ 618 West Chang'an Street, Chang'an District, Xi'an City 710121, China \\ ${ }^{3}$ School of Creative Arts and Engineering, Staffordshire University, \\ College Road, University Quarter, Stoke-on-Trent Staffordshire ST4 2DE, UK
}

(Received July 15, 2019; accepted October 18, 2019)

Keywords: fall detection, eigenvalues, support vector, SVM fall model

The use of a single method of acceleration threshold discrimination cannot fully characterize the change in human fall behavior, which can easily result in misjudgment. In this paper, we propose a human fall detection algorithm that combines human posture, support vector machine (SVM), and quadratic threshold decision. Firstly, a large number of human posture data are collected through a six-axis inertial measurement module (MPU6050). A fall detection model is established through filtering preprocessing, eigenvalue extraction, classification, and SVM training. Secondly, a first-level threshold determination is performed through a wearable wristband device. When a suspected fall occurs, six eigenvalues will be captured and uploaded to a cloud platform to trigger second-level SVM fall judgments. By matching the eigenvalues with the fall detection model, it can be determined accurately whether a fall has taken place. The experimental results show that the fall detection has a recognition rate of $92.2 \%$, a false rate of $3.593 \%$, and missing rate of $2.187 \%$, which can better distinguish other nonfall actions.

\section{Introduction}

Fall detection systems can be classified into three types based on video images, physical environment, and wearable devices. ${ }^{(1)}$ The video-image-based system can achieve accurate fall detection and recognition, but it requires a camera to be fixed in a room, thus limiting user's activities. In addition, this system is mostly not accepted by users because of personal privacy. The fall detection system based on the physical environment, such as a plantar pressure sensor or an infrared sensor, is for indoor use only. Considering the limited monitoring range and high installation cost, it is only used in specific scenarios and thus is not accepted by the public. ${ }^{(2)}$ However, the fall detection system based on wearable devices has the advantage, that is, it can be used both indoor and outdoor, ${ }^{(3)}$ with no limit on the user's behaviors and no violation of privacy. ${ }^{(4)}$ Its alarm mode is relatively flexible, and its low price is suitable for widespread

*Corresponding author: e-mail: qujunsuo@xupt.edu.cn https://doi.org/10.18494/SAM.2020.2527 
promotion, but the recognition rate of fall behavior is not satisfactory, and different devices need to have corresponding fall detection algorithms.

In this paper, we propose a human fall detection algorithm based on multiple-threshold comprehensive judgment and design a fall detection wearable device with STM32 as a hardware platform, MPU6050 as a sampler, and SIM808 as a communication positioning module, equipped with a fall detection bracelet. It is suitable for both indoor and outdoor, which determines the fall state through two levels, and can trigger an alarm automatically. It is also efficient, accurate, and easy to wear. It has advantages of small size, simplicity, and stable operation. $^{(5)}$

\section{System Design of Fall Detection Bracelet}

\subsection{Overall design of the system}

The scheme consists of three parts: a wearable device, a cloud platform, and a mobile application. The overall design of the system is shown in Fig. 1.

The wearable device collects human body posture information, conducts first-level fall detection, and uploads global positioning system (GPS) information to a cloud platform periodically. A guardian can monitor a user's current location and historical trajectory through the application. When a fall is detected, the buzzer on the bracelet will sound an alarm immediately and a user's suspected fall message will be sent to the guardian. At the same time, the secondary fall detection with the support vector machine (SVM) will be triggered. The platform judges the fall accurately by comparing the fall message with fall detection model. If it is true, the platform sends a confirmation message to the wearable device and dials the

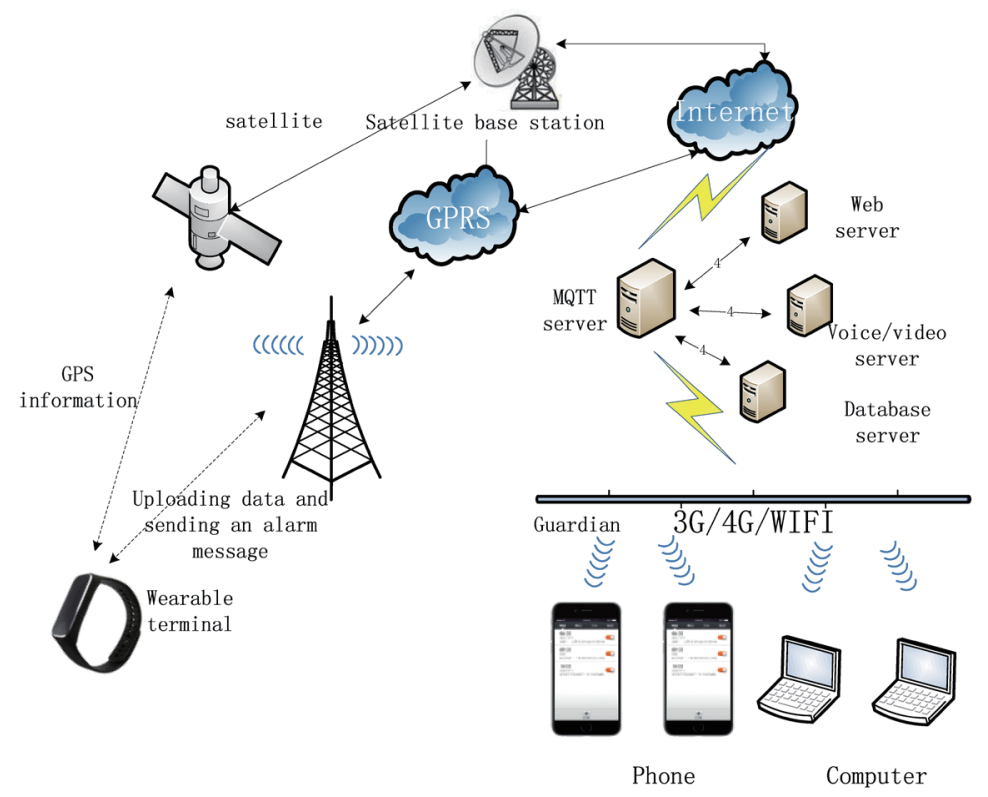

Fig. 1. (Color online) Overall design of the system. 
guardian's phone or emergency center, so that the user will have medical treatment on time. ${ }^{(6)}$ When the wearable device triggers an alarm, the user has a choice to cancel the alarm by pressing the cancellation button.

\subsection{Hardware design}

The hardware of the fall detection bracelet is composed of an STM32F103 microcontroller, an MPU6050 six-axis inertial sensor module, an SIM808 communication positioning module, a button module, a power management module, and a buzzer. The block diagram of the bracelet is shown in Fig. 2. This bracelet is powered by a $3.7 \mathrm{~V}$ lithium battery, which is small in size and large in capacity, and with a long standby time.

\subsection{Software design}

The software of the fall detection system can be divided into three functional modules: fall detection, fall alarm, and remote monitoring. The system software flow chart is shown in Fig. 3.

\section{Research on Human Fall Behavior}

Human activity patterns can be divided into two categories. One category is composed of daily activities, which can be divided into basic behaviors, similar to fall behaviors. ${ }^{(7)}$ Basic behaviors include standing, sitting, lying, walking, applauding, and waving. Similarly, fall behaviors include sitting down, lying down, squatting, jumping, and going up and down the stairs. The other category is composed of fall behaviors, such as fall forward, fall backward, fall to the left, and fall to the right. ${ }^{(8)}$

\subsection{Fall behavior}

Fall behavior can be simply understood as the human body being affected by physiology, disease, environment, and so forth. The body falls to the ground suddenly or to a low plane involuntarily. The fall behavior can be divided into three phases from the beginning to the end, namely, the unbalance, weight loss, and collision phases. ${ }^{(9)}$

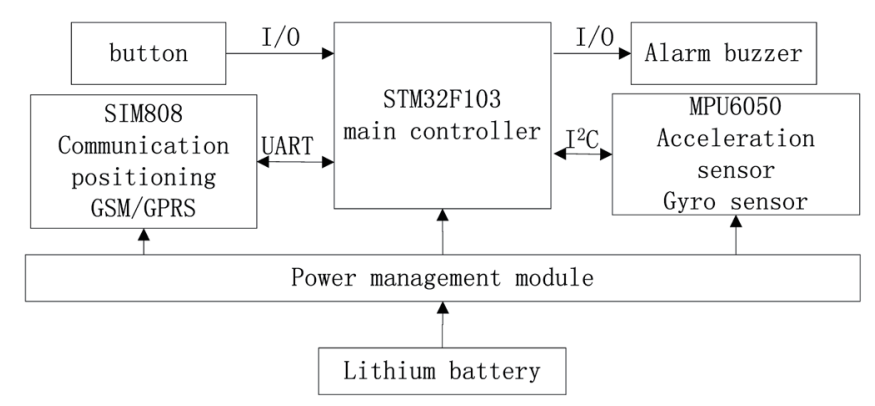

Fig. 2. Hardware design block diagram. 


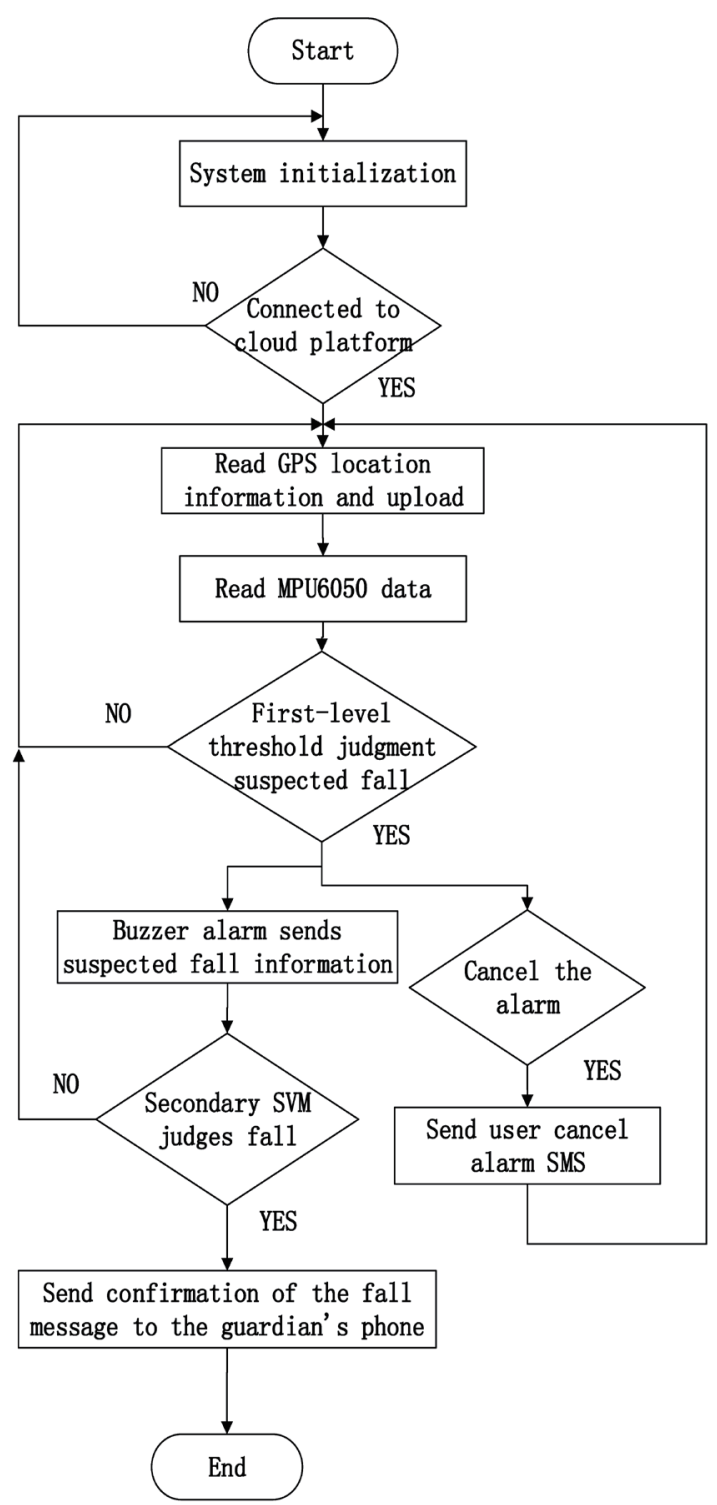

Fig. 3. Software design flow chart.

\subsection{Analysis of fall model}

In this paper, the wearable device is used for fall detection, so the energy change of the human body, the angle change of the body, and the change of the movement of the arm are all important reference factors. Establishing a suitable and accurate human posture model is the basis for the analysis of the fall behavior of the human body.

When the body falls, the most obvious change takes place in the weight loss and collision phases. During this period, the human body's energy will change considerably owing to weightlessness or violent collision with the ground. If the human body is standing in a scene, 
the front and back of the human body are set to the $x$-axis, the sides of the human body are set to the $y$-axis, and the direction perpendicular to the human body is set to the $z$-axis. ${ }^{(10)}$ Referring to the human body's three-dimensional coordinate model, we set the lost energy to be $E_{c}$. The energy loss can be calculated as

$$
E_{c} \approx \int_{T}\left(\left|F_{x}\right|+\left|F_{y}\right|+\left|F_{z}\right|\right) d t
$$

In Eq. (1), $F_{x}, F_{y}$, and $F_{z}$ represent the magnitudes of the different component forces in the $x$-axis, $y$-axis, and $z$-axis directions, respectively, in the weight loss or collision phase, whereas $T$ is the time from the unbalance phase to the completion of the collision phase. According to Newton's second law of motion, if the force is $F$, the acceleration is a, and the mass of an object is $m$. Newton's second law of motion can be expressed as ${ }^{(11)}$

$$
\vec{F}=m \cdot \vec{a}
$$

Expanded from the $x$-, $y$-, and $z$-axes, it can be expressed as

$$
F_{x}=m \cdot a_{x}, F_{y}=m \cdot a_{y}, F_{z}=m \cdot a_{z} \cdot
$$

It can be inferred from Eqs. (1) and (3) that the energy loss is proportional to the amount of triaxial acceleration change during the fall, namely,

$$
E_{c} \propto \int_{T}\left(\left|a_{x}\right|+\left|a_{y}\right|+\left|a_{z}\right|\right) d t
$$

It is inferred from the above theory that the fall behavior can be transformed into the collection, analysis, and judgment of the special amount of collected data produced by the body change. The data on triaxial acceleration and three-axis angular velocity are collected by the MPU6050 sensor module. ${ }^{(12)}$ By analyzing and extracting the original data, the changes in human body energy and angle can be obtained during the fall, so as to accurately determine the fall behavior.

In this study, the square root of the added triaxial accelerations is set to the judgment unit to eliminate the effect of the direction uncertainty. ${ }^{(13)}$ With $A c c$ set as a resultant acceleration, the expression is

$$
A c c=\sqrt{A_{x}^{2}+A_{y}^{2}+A_{z}^{2}},
$$

where $A_{x}, A_{y}$, and $A_{z}$ represent the acceleration data of the $x$-, $y$-, and $z$-axes, respectively, and the data are collected by the MPU6050 six-axis inertial measurement module. Acc is termed as resultant acceleration. Thus, the collected data can be used for the analysis of human daily activities and fall behavior. 


\subsection{Data analysis of typical behaviors}

The resultant acceleration and human activities are inseparable, according to the results of the analysis of the fall model. With the resultant acceleration as a basis for determination, researchers can accurately distinguish between normal movements and the fall behavior. Two boys and one girl with different heights and weights were selected in the experiment, and the acceleration data of typical human activities were collected. Typical activity behaviors include walking slowly, standing still, sitting down, lying down, going up and down the stairs, falling, and so on. In the experiment, the three-axis acceleration data is collected by the MPU6050 sensor module, and the three-axis acceleration data is processed into the resultant acceleration and output by the serial port assistant. The typical activity behaviors of the MATLAB simulation are shown in Fig. 4.

From the results of the analysis of different human activities, we determined the ranges of daily activities and fall behaviors of the human body, as shown in Table 1.

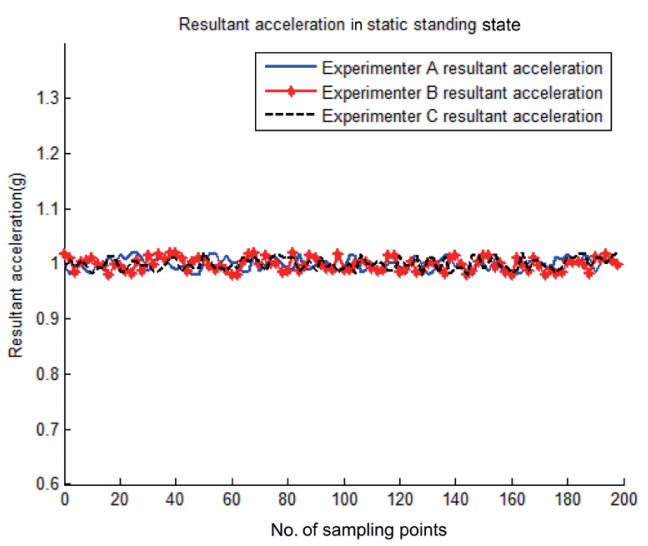

(a)

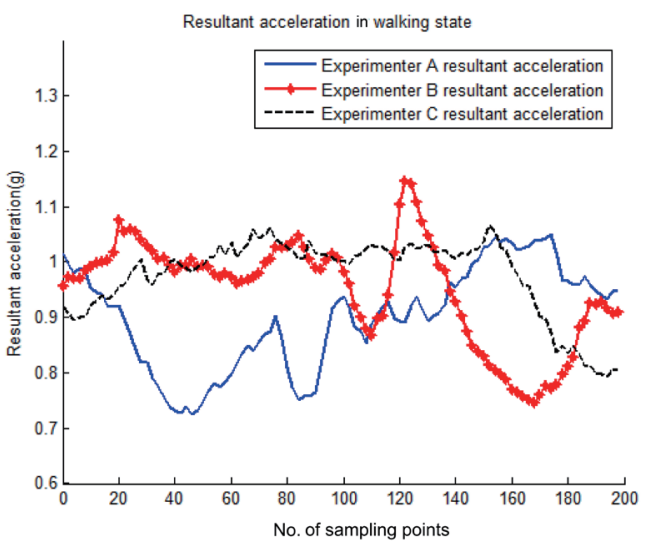

(b)

Fig. 4. (Color online) Simulation diagram of typical activity behaviors. (a) Resultant acceleration curve in static standing state and (b) curve of resultant acceleration in walking state.

Table 1

Synthetic acceleration range statistics of various active modes of human body.

\begin{tabular}{lc}
\hline Human activity & Synthetic acceleration $A c c$ range $(g)$ \\
\hline Standing & $0.967544-1.030999$ \\
Walking & $0.732901-1.145872$ \\
Sitting down & $0.695462-1.927811$ \\
Lying down & $0.513798-1.905217$ \\
Squatting & $0.522330-1.566881$ \\
Standing up & $0.606632-1.354742$ \\
Going up the stairs & $0.689217-1.331505$ \\
Going down the stairs & $0.483373-1.917394$ \\
Fall forward & $0.329170-4.373055$ \\
Fall backward & $0.294463-5.175019$ \\
Fall to the left & $0.306855-4.341224$ \\
Fall to the right & $0.308100-5.142192$ \\
Applauding & $0.292725-1.992981$ \\
Waving & $0.301599-1.990967$ \\
\hline
\end{tabular}


It can be seen from the above table that the synthetic acceleration range of daily activities is from 0.483373 to $1.992981 \mathrm{~g}$, and that of fall behaviors is from 0.294463 to $5.175019 \mathrm{~g}$. There is an obvious division between daily activities and fall behaviors, so the acceleration can be used as the eigenvalue of the first-level threshold determination.

\subsection{Analysis of fall behavior feature}

Unlike the traditional fall detection device fixed on the head, waist, and back, the wrist-type fall detection device can focus on the tilt angle of the human body and the angular velocities of the three axes. ${ }^{(14)}$ Therefore, the eigenvalue and feature vector should be chosen and further analyzed. The following six signal characteristics are chosen for fall detection.

1. Acceleration intensity vector (signal magnitude vector, SMV)

$$
S M V=\sqrt{A_{x}^{2}+A_{y}^{2}+A_{z}^{2}}
$$

$S M V$ is actually the resultant acceleration $A c c$. The maximum and minimum Acc values are the eigenvalues of the fall detection.

In the process of human fall, from the unbalanced phase to the weightlessness period, the human body loses balance and the body is in a free fall stage. The resultant acceleration of the human body will have a decreasing change, ${ }^{(15)}$ and the minimum resultant acceleration is set to $S M V_{\min }$. It is selected as the first eigenvalue of the behavior.

The third stage of the fall behavior is the collision stage. In this stage, the human body will have a strong impact with the ground. When the human body hits the ground, the speed of the human body is zero, and the maximum acceleration of the body is set to $S M V_{\max }$. It reflects the violent degree of impact between the human body and the ground, and is selected as the second eigenvalue of the behavior.

2. Signal amplitude area (SMA)

$$
S M A=\frac{1}{T}\left(\int_{0}^{T}\left|A_{x}\right| d t+\int_{0}^{T}\left|A_{y}\right| d t+\int_{0}^{T}\left|A_{z}\right| d t\right)
$$

In Eq. (7), $T$ represents the time from the start of the weight loss phase to the end of the collision phase.

SMA is calculated using the absolute value of the triaxial acceleration as a basic parameter, which can intuitively reflect the intensity of the human motion state, and is selected as the third eigenvalue of the behavior.

3. Gyro resultant angular velocity (Gry)

$$
G r y=\sqrt{G_{x}^{2}+G_{y}^{2}+G_{z}^{2}}
$$

In Eq. (8), $G_{x}, G_{y}$, and $G_{z}$ represent the angular velocities of the $x$-, $y$-, and $z$-axes, respectively, and can be acquired using the gyro sensor of the MPU6050 sensor module. Gry can reflect the 
severity of the wrist or body rotation when the human body is active. ${ }^{(16)}$ However, the angular velocity avoids the complexity of different axial directions effectively. The maximum Gry is selected as the fourth eigenvalue of the behavior.

4. Time of weightlessness (TWL)

$$
T W L=T_{L}-T_{0}
$$

$T W L$ is defined as the time from the unbalance phase to the end of the weight loss phase. ${ }^{(17)}$ $T_{0}$ is the first point time when the free fall begins. $T_{L}$ is the last point time in the free fall movement. The weight loss duration is selected as the fifth eigenvalue of the behavior.

5. Time of impact (TIM)

$$
T I M=T_{S V M_{\max }}-T_{S V M_{\min }}
$$

TIM is defined as the period from the start of the impact phase to the end of the impact phase. It is selected as the sixth eigenvalue.

\section{Fall Detection Algorithm Based on Quadratic Decision}

In this study, the SVM classifier method is used to obtain the suspected fall signal data from the first input first output (FIFO), and six of the eigenvalues are extracted. By matching with the fall detection model, ${ }^{(18)}$ an accurate second judgment result can be obtained.

We use LIBSVM as the training fall data. LIBSVM is a simple, easy-to-use, fast, and effective SVM pattern recognition and regression software package developed by Lin Zhiren of Taiwan University. A large number of human posture data should be acquired through the MPU6050 sensor module. The data has been saved after preprocessing and eigenvalue processing, and then the six eigenvalues have been edited into a file according to the input format of the SVM, made into corresponding training sets, and finally, the fall detection model is trained. In this work, we use 4000 sample data for training and 80 data for testing.

Next, the bracelet needs to realize the judgment of a suspected fall. When the detected resultant acceleration is larger than the threshold value, the suspected fall is determined. At the same time, the secondary SVM fall detection will be triggered, which requires the bracelet to upload the data carrying fall features to the platform, and then through comparison with the fall detection model, an accurate determination can be made.

The algorithm detection steps are as follows.

1. Suspected fall determination. The wristband detects the magnitude of the human body resultant acceleration in real time. When the detected acceleration is greater than the set one-level threshold acceleration, it is determined that a suspected fall occurs.

2. Extract eigenvalues. Six eigenvalues are extracted from the suspected fall signal segment.

3. Second fall determination. The bracelet uploads the processed fall data to the platform and compares it with the fall detection model to accurately determine whether it falls. if it is determined to be a fall, the fall is confirmed. Otherwise, it is determined to be a false alarm. Figure 5 shows the algorithm flow. 


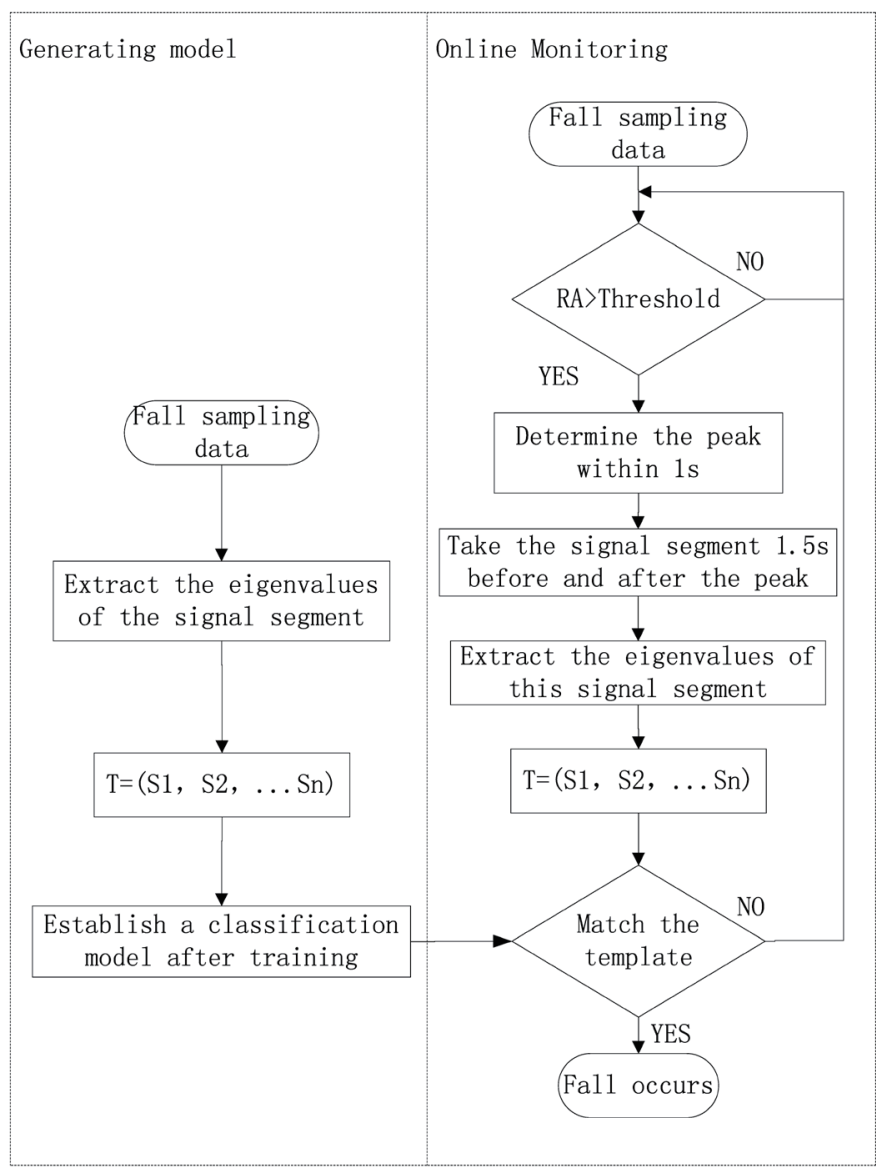

Fig. 5. Flow chart of the fall detection algorithm.

\section{Experiment and Analysis}

The fall detection bracelet prototype with a diameter of $80 \mathrm{~mm}$ is shown in Fig. 6 .

\subsection{Threshold selection experiment}

One male and one female were selected to contribute 10 sets of fall behavior data and 20 groups of normal movements, respectively. The test results are shown in Table 2. Among them, $g=9.8 \mathrm{~N} / \mathrm{kg}$.

From the above table, to ensure a lower missing rate, $3.2 \mathrm{~g}$ is selected in this experiment as the first-level decision threshold. It can be seen from the above table that when the threshold is between 2.8 and $3.6 \mathrm{~g}$, the first-level threshold judgment has almost no false report of a fall. When the threshold is between 3.8 and $4.4 \mathrm{~g}$, there is a false report. According to the experimental results, there are more false positives in the first-level judgment between 2.8 and $3.2 \mathrm{~g}$, so it is more reasonable to select $3.2-3.6 \mathrm{~g}$. To ensure a lower false negative rate, $3.2 \mathrm{~g}$ is selected in this experiment as the first-level decision threshold. 


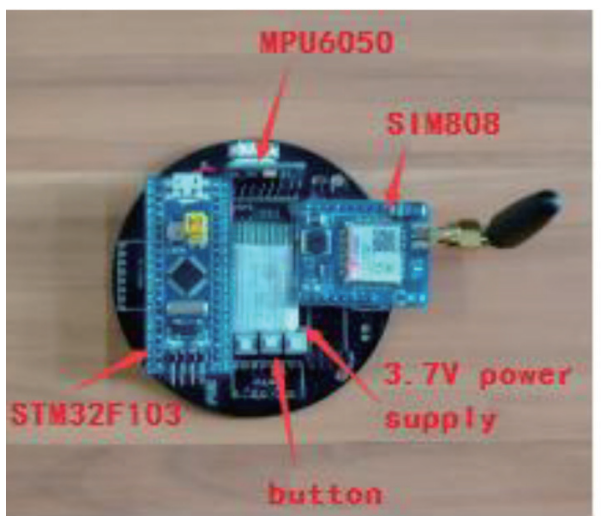

Fig. 6. (Color online) Fall detection bracelet prototype.

Table 2

Comparison of the first- and second-level thresholds.

\begin{tabular}{|c|c|c|c|c|c|}
\hline & \multicolumn{2}{|c|}{ First-level threshold determination } & \multicolumn{2}{|c|}{ Second-level SVM determination } & \multirow{2}{*}{$\begin{array}{l}\text { Number of } \\
\text { missing falls }\end{array}$} \\
\hline $\begin{array}{l}\text { Threshold } \\
\text { setting }(g)\end{array}$ & $\begin{array}{c}\text { Determining } \\
\text { normal behavior }\end{array}$ & $\begin{array}{c}\text { Determining fall } \\
\text { behavior }\end{array}$ & $\begin{array}{l}\text { Determining } \\
\text { daily behavior }\end{array}$ & $\begin{array}{c}\text { Determining fall } \\
\text { behavior }\end{array}$ & \\
\hline 2.8 & 16 & 14 & 2 & 12 & 0 \\
\hline 3.0 & 15 & 15 & 3 & 12 & 0 \\
\hline 3.2 & 18 & 12 & 3 & 9 & 1 \\
\hline 3.4 & 18 & 12 & 1 & 11 & 0 \\
\hline 3.6 & 19 & 11 & 0 & 11 & 0 \\
\hline 3.8 & 21 & 9 & 0 & 9 & 1 \\
\hline 4.0 & 23 & 7 & 2 & 5 & 5 \\
\hline 4.2 & 23 & 7 & 0 & 7 & 3 \\
\hline 4.4 & 24 & 6 & 0 & 6 & 4 \\
\hline
\end{tabular}

\subsection{Experiment on the algorithm effectiveness}

After selecting the first-level threshold values, we will use the sample data set collected via the experiment to evaluate the effectiveness of the fall detection algorithm completely through three indicators, namely, recognition rate (CR), missing rate (MR), and false rate (FR).

Suppose $n$ experiments are performed, in which the normal behaviors are performed $a$ times and the fall behavior is performed $n-a$ times. The frequency of triggering the SVM secondary fall determination is $p$, and the number of times the SVM accurately identifies the fall and secondary acknowledgement alarm is $q$.

Recognition rate $[C R=q / n$ or $C R=(n-q) / n]$ : The fall detection bracelet can correctly distinguish the probabilities of normal and fall behaviors. The recognition rate for normal behaviors is $C R=(n-q) / n$, whereas that for the fall behavior is $C R=q / n$.

Missing rate $[M R=(n-a-p) / n]$ : The probability that a fall behavior that is not recognized by the first-level threshold algorithm occurs.

False rate $(F R=p / a)$ : The probability that a primary threshold algorithm misinterprets a daily activity as a fall behavior. 
In a fall detection system, the missing rate should be reduced to 0 as much as possible to better ensure the safety of the user groups. At the same time, the higher the recognition rate, the higher the effectiveness of the fall detection algorithm. In addition to a comparatively low false rate, the user will have a satisfactory experience with the product.

In the experiment to verify validity, eight experimenters respectively simulated ten groups of eight normal behaviors including standing, walking, sitting down, lying down, squatting, standing up, going up the stairs, and going down the stairs, and participated in a fall detection experiment. In the normal behavior testing, the recognition and false rates were used as evaluation criteria. Results are shown in Table 3.

The same group of experimenters simulated ten groups of falling, including fall forward, fall backward, fall to the left, and fall to the right. The experimental results based on the criteria of the recognition and missing rates are shown in Table 4.

From Tables 3 and 4, the fall detection bracelet has a recognition rate of $97.8125 \%$ and a false rate of $3.59375 \%$ in the normal behavior detection. In the fall behavior detection, the system recognition rate reaches $92.5 \%$ and the missing rate is $2.1875 \%$. The reason for the high missing rate of leftward fall is that the wristband is worn on the right side of the tester, and the left hand has a buffering action to push the body up over the ground, which reduces the acceleration. The missing report of the forward fall lies in the reduced acceleration caused by the experimenters' knee-buffering action.

Table 3

Experimental evaluation results of normal behaviors.

\begin{tabular}{|c|c|c|c|c|c|}
\hline Event & $\begin{array}{c}\text { Number of } \\
\text { experiments }\end{array}$ & $\begin{array}{l}\text { Number of alarms } \\
\text { at the first level }\end{array}$ & $\begin{array}{c}\text { Actual number of } \\
\text { alarms }\end{array}$ & $\begin{array}{c}\text { Recognition rate } \\
(\%)\end{array}$ & $\begin{array}{c}\text { False rate } \\
(\%)\end{array}$ \\
\hline Standing & 80 & 0 & 0 & 100 & 0 \\
\hline Walking & 80 & 3 & 1 & 98.75 & 3.75 \\
\hline Sitting down & 80 & 5 & 3 & 96.25 & 6.25 \\
\hline Lying down & 80 & 2 & 0 & 100 & 2.5 \\
\hline Squatting & 80 & 8 & 6 & 92.5 & 10 \\
\hline Standing up & 80 & 3 & 2 & 97.5 & 3.75 \\
\hline Going up the stairs & 80 & 0 & 0 & 100 & 0 \\
\hline Going down the stairs & 80 & 2 & 2 & 97.5 & 2.5 \\
\hline Sum & 640 & 23 & 14 & 97.8125 & 3.59375 \\
\hline
\end{tabular}

Table 4

Experimental evaluation results of fall behaviors.

\begin{tabular}{lccccc}
\hline Event & $\begin{array}{c}\text { Number of } \\
\text { experiments }\end{array}$ & $\begin{array}{c}\text { Number of alarms } \\
\text { at the first level }\end{array}$ & $\begin{array}{c}\text { Actual number of } \\
\text { alarms }\end{array}$ & $\begin{array}{c}\text { Recognition rate } \\
(\%)\end{array}$ & False rate (\%) \\
\hline Fall forward & 80 & 78 & 73 & 91.25 & 2.5 \\
Fall backward & 80 & 80 & 78 & 97.5 & 0 \\
Fall to the left & 80 & 75 & 70 & 87.5 & 6.25 \\
Fall to the right & 80 & 80 & 75 & 93.75 & 0 \\
Sum & 320 & 313 & 296 & 92.5 & 2.1875 \\
\hline
\end{tabular}




\section{Conclusions}

In this paper, we present a human fall detection algorithm that combines human posture, SVM, and quadratic threshold decision. A fall detection model is established and a fall detection bracelet has been designed to realize fall detection, fall alarm, remote monitoring, and other functions. The experimental results show that the fall detection bracelet, with a recognition rate of $92.2 \%$, a false rate of $3.593 \%$, and an under-reporting rate of $2.187 \%$, can well distinguish falls from other nonfall actions.

\section{Acknowledgments}

This research was supported in part by grants from the National Natural Science Foundation of China under Grant No. 51875457, the International Cooperation and Exchange Program of Shaanxi Province under Grant No. 2018KW-026, the Natural Science Foundation of Shaanxi Province under Grant Nos. 2018JM6120 and 2019JM-606, and Xi'an Science and Technology Projects under Grant No. 201805040YD18CG24(6).

\section{References}

1 Z. Huang, Y. Liu, and Y. Fang: 2018 4th Int. Conf. Universal Village (UV) (IEEE, 2018) 41. https://doi. org/10.1109/UV.2018.8642130.

2 Y. Zheng, N. Bao, L. Xu, X. Lin, T. Huang, and Y. Dou: Chin. J. Med. Phys. 31 (2014) 5071. https://doi. org/10.3969/j.issn.1005-202X.2014.04.021

3 S. Agrawal, R. Tripathi, and A. Jalal: 2017 8th Int. Conf. Computing, Communication, and Networking Technologies (ICCCNT) (IEEE, 2017) 1. https://doi.org/10.1109/ICCCNT.2017.8203923

4 C. Yang, C. Chen, N. Wang, Z. Ju, J. Fu, and M. Wang: IEEE Trans. Cognit. Dev. Syst. 11 (2019) 281. https:// doi.org/10.1109/TCDS.2018.2866477

5 Z. Wu, L. Cao, K. Wang, and Q. Lin: Comput. Eng. Des. 34 (2014) 1465. https://doi.org/10.16208/ j.issn1000-7024.2014.04.070

6 W. Xu, W. Meng, and L. Zeng: Autom. Instrum. 31 (2016) 35. https://doi.org/10.19557/ j.cnki.1001-9944.2016.11.009

7 C. Yang, J. Luo, C. Liu, M. Li, and S. Dai: IEEE Trans. Autom. Sci. Eng. (Early Access) (IEEE, 2018$) 1$. https://doi.org/10.1109/TASE.2018.2874454

8 M. Arif, M. Bilal, and A. Kattan: J. Med. Syst. 38 (2014) 1. https://doi.org/10.1007/s10916-014-0095-0

9 L. Ren and Y. Peng: Syst. Rev. 7 (2019) 77702. https://doi.org/10.1109/ACCESS.2019.2922708

10 L. Chen, Hanna, and L. Chen: J. Xi'an University of Posts and Telecommunication 5 (2016) 43. https://doi. org/10.13682/j.issn.2095-6533.2016.05.009

11 G. Yang and W. Wang: Comput. Meas. Control 24 (2016) 56. https://doi.org/10.16526/j.cnki.11-4762/ tp.2016.08.015

12 L. Pei, P. Jiang, and G. Yan: Opt. Precis. Eng. 25 (2017) 183. https://doi.org/10.3788/OPE.20172501.0182.

13 R. Sun, S. Ma, and S. Wang: Mod. Electron. Tech. 40 (2017) 14. https://doi.org/10.16652/j.issn.1004373x.2017.22.004

14 J. Zheng and T. Wu: Acad. Mil. Med. Sci. 11 (2008) 140. https://doi.org/10.1007/s11517-007-0221-y

15 Y.Cheng, D. Li, and X. Zhang: Electron. Des. Eng. 24 (2016) 181. https://doi.org/10.14022/j.cnki. dzsjgc.2016.14.055

16 J. Liang, H. Du, and Y. Yao: Comput. Eng. Software 37 (2016) 21. https://doi.org/10.3969/j.issn.10036970.2016.07.005

17 F. Hussain and F. Hussain: IEEE Sens. J. 19 (2019) 4528. https://doi.org/10.1109/JSEN.2019.2898891

18 H. Kao, J. Hung, and C. Huang: 2017 Int. Conf. Applied System Innovation (IEEE, 2017) 13. https://doi. org/10.1109/ICASI.2017.7988446 


\section{About the Authors}

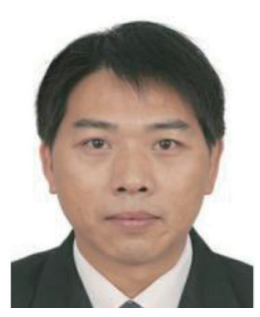

Junsuo Qu received his B.S. degree in telecommunication engineering from Chongqing University of Posts \& Telecommunications, Chongqing, China, in 1991, and his M.S. degree in communication and information systems from Xidian University, Xi'an, China, in 1998. He is a full professor of the School of Automation of Xi'an University of Posts \& Telecommunications and a member of the China Institute of Communications. He is the director of Xi'an Key Laboratory of Advanced Control and Intelligent Process. He is leading an IoT research team in the School of Automation.

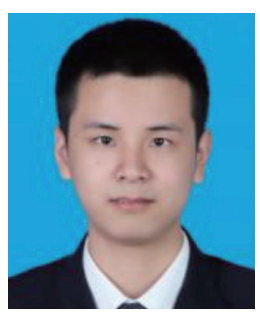

Chen $\mathbf{W u}$ received his B.S. degree in communication engineering from Xi'an University of Posts \& Telecommunications, Shaan Xi, China, in 2016. He is now studying for a master's degree in Xi'an University of Posts \& Telecommunications. His main research topic is the technology and application of Internet of Things.

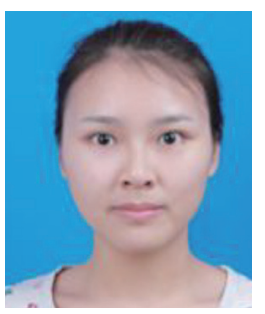

Qian Li received her B.S. degree in electronic science and technology from Baoji University of Arts and Sciences, Bao ji, Shaanxi, China, in 2018. She is now studying for a master's degree in Xi'an University of Posts \& Telecommunications. Her research interests are in Internet of Things technology and applications.

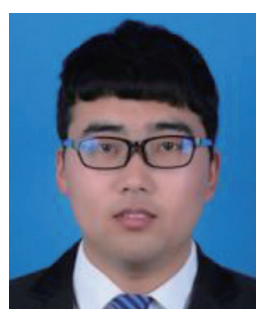

Ting Wang received his B.S. degree in electronic information science and technology from Xi'an University of Posts \& Telecommunications, Xi'an, China, in 2016. He is now studying for a master's degree in Xi'an University of Posts \& Telecommunications. His main research topic is the technology and application of Internet of Things.

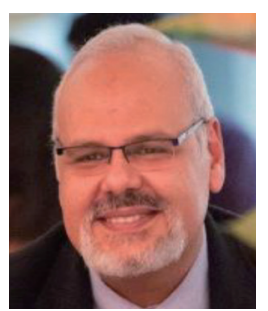

Abdel Hamid Soliman received his Ph.D. degree in image processing and pattern recognition from Staffordshire University, and his master's degree in information technology from Alexandria University. He is a core member of the Staffordshire University Mobile Fusion (MF) Applied Research Centre. $\mathrm{He}$ is leading the smart system research team for smart city applications in the Department of Engineering and working now to establish a renewable energy and smart system center in the school, aiming to provide the latest technologies in the field to serve research purposes. 